\title{
Low energy effective theory on a regularized brane in six-dimensional flux compactifications
}

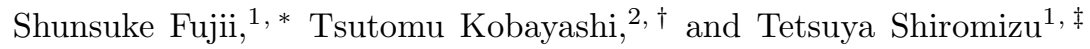 \\ ${ }^{1}$ Department of Physics, Tokyo Institute of Technology, Tokyo 152-8551, Japan \\ ${ }^{2}$ Department of Physics, Waseda University, Okubo 3-4-1, Shinjuku, Tokyo 169-8555, Japan
}

\begin{abstract}
Conical brane singularities in six-dimensional flux compactification models can be resolved by introducing cylindrical codimension-one branes with regular caps instead of 3-branes (a la KaluzaKlein braneworlds with fluxes). In this paper, we consider such a regularized braneworld with axial symmetry in six-dimensional Einstein-Maxwell theory. We derive a low energy effective theory on the regularized brane by employing the gradient expansion approach, and show that standard four-dimensional Einstein gravity is recovered at low energies. Our effective equations extend to the nonlinear gravity regime, implying that conventional cosmology can be reproduced in the regularized braneworld.
\end{abstract}

PACS numbers: $04.50 .+\mathrm{h}$

\section{INTRODUCTION}

Recent developments in string theory provide us with a remarkably new picture of our universe, in which our universe is described by the motion of a 3-brane in a higher dimensional spacetime. In addition, it has been suggested that extra dimensions warped due to self-gravity of branes may play a key role in addressing the gauge hierarchy problem. A simple toy model of warped braneworlds was proposed by Randall and Sundrum [1] and its various consequences has been studied extensively [2]. The idea of warped extra dimensions is plugged in warped flux compactifications, in which reliable inflation models based on string theory has been constructed [3] (see Ref. [4] for a review).

While the five-dimensional (5D) Randall-Sundrum-type models [1] are simple enough, offering us a feasible setup for studying the behavior of gravity in braneworlds, brane models in six or higher dimensions have a much richer structure such as flux-stabilized compactifications. In particular, codimension-two braneworlds with football-shaped extra dimensions attracted much attention initially, due to their potential mechanism for solving the cosmological constant problem [ 5, [6, 7]. However, a higher codimension brane develops a conical or worse singularity once taking into account its self-gravity, which hampers a substantial analysis of higher dimensional braneworlds. Thus, it is necessary to regularize higher codimension branes in a well-controllable way to construct reasonable models of higher dimensional braneworlds. One of the regularization procedure is to replace a higher codimension defect with a codimension-one brane, whose extra dimensions are compactified in the traditional Kaluza-Klein manner. This was done in an specific model of $6 \mathrm{D}$ flux compactifications in $[8,[9,10]$. This type of models may be regarded as a variation of Kaluza-Klein/hybrid braneworlds [11, 12, 13, 14, 15]. Only pure tension, gravitational shockwaves, and black holes have so far been known to be accommodated on codimension-two branes [16], but with this regularization scheme one can put arbitrary energy-momentum tensor on the branes. Performing perturbation analyses, it has been shown that weak gravity sourced by arbitrary matter on the regularized brane reproduces standard 4D one 8, 17].

In this paper we further investigate the behavior of gravity on the regularized brane in the $6 \mathrm{D}$ flux compactification models, generalizing the previous result to cover nonlinear gravity. We keep in mind the bulk-brane configuration of [8, 9] , but start with a more general metric ansatz. Employing the gradient expansion approach, we solve the $6 \mathrm{D}$ Einstein-Maxwell equations to derive an effective theory on the brane. The gradient expansion approach in braneworlds is based on the $(4+1)$-decomposition of the bulk spacetime [18] and was originally developed in the context of the 5D Randall-Sundrum model [19, 20]. The gradient expansion has been widely used in various brane models (e.g., 21]). Recently, along the direction of the regularized braneworlds, the geometrical projection approach of [18] has been generalized to non- $Z_{2}$-symmetric models in an arbitrary number of dimensions [22].

The plan of the paper is as follows. In the next section we perform the $(5+1)$-decomposition of $6 \mathrm{D}$ spacetime. In Sec. III the evolution equations along one of the extra direction are solved by invoking the gradient expansion approach up to first order. We explain how conical singularities in the bulk are regularized by introducing extended

\footnotetext{
*Email: fujii" at"th.phys.titech.ac.jp

†Email: tsutomu" at" gravity.phys.waseda.ac.jp

‡Email: shiromizu" at"phys.titech.ac.jp
} 
4-branes in Sec. IV] In Sec. V] we derive the effective theory on the regularized branes using the result of Sec. III In Sec. VI we draw our conclusions.

\section{BASIC EQUATIONS}

The $6 \mathrm{D}$ action we consider is

$$
S=\int d^{6} x \sqrt{-g}\left[\frac{M^{4}}{2}\left(R-\frac{1}{L_{I}^{2}}\right)-\frac{1}{4} F^{2}\right]
$$

where $F^{2}=F_{M N} F^{M N}$ and $F_{M N}=\partial_{M} A_{N}-\partial_{N} A_{M}$ is the field strength of the $U(1)$ gauge field $A_{M}$. $M$ is the fundamental scale of gravity and $1 / 2 L_{I}^{2}$ is the cosmological constant. Branes will be added in Sec. IV and for the time being we will concentrate on solving the $6 \mathrm{D}$ geometry. We assume the axisymmetric metric ansatz in the form of

$$
g_{M N} d x^{M} d x^{N}=L_{I}^{2} \frac{d y^{2}}{f(y)}+\ell^{2} e^{2 \psi(x, y)} f(y) d \theta^{2}+2 \ell b_{\mu}(x, y) d \theta d x^{\mu}+\tilde{g}_{\mu \nu}(x, y) d x^{\mu} d x^{\nu}
$$

with

$$
\tilde{g}_{\mu \nu}=a^{2}(y) \tilde{h}_{\mu \nu}(x, y)
$$

The capital Latin indices numerate the $6 \mathrm{D}$ coordinates, while the Greek indices are restricted to the $4 \mathrm{D}$ coordinates.

We write the 6D Einstein equations in the form of the evolution equations along the $y$-direction and the constraint equations. The evolution equations are

$$
n^{y} \partial_{y} K_{\hat{\mu}}^{\hat{\nu}}+\hat{K} K_{\hat{\mu}}^{\hat{\nu}}={ }^{5} R_{\hat{\mu}}^{\hat{\nu}}-\frac{1}{4 L_{I}^{2}} \delta_{\hat{\mu}}^{\hat{\nu}}-\frac{1}{M^{4}}\left(F_{\hat{\mu} M} F^{\hat{\nu} M}-\frac{1}{8} \delta_{\hat{\mu}}^{\hat{\nu}} F^{2}\right),
$$

where $n^{y}=\sqrt{f} / L_{I}, K_{\hat{\mu}}^{\hat{\nu}}$ is the extrinsic curvature of $y=$ constant hypersurfaces, and $\hat{K}$ is its $5 \mathrm{D}$ trace. ${ }^{5} R_{\hat{\mu}}^{\hat{\nu}}$ is the 5D Ricci tensor. Here, $\hat{\mu}=\mu$ and $\theta$. The Hamiltonian constraint is

$$
{ }^{5} R+K_{\hat{\mu}}^{\hat{\nu}} K_{\hat{\nu}}^{\hat{\mu}}-\hat{K}^{2}=\frac{1}{L_{I}^{2}}-\frac{2}{M^{4}}\left(F_{y M} F^{y M}-\frac{1}{4} F^{2}\right) .
$$

and the momentum constraints are

$$
{ }^{5} D_{\hat{\nu}}\left(K_{\hat{\mu}}^{\hat{\nu}}-\delta_{\hat{\mu}}^{\hat{\nu}} \hat{K}\right)=\frac{1}{M^{4}} F_{\hat{\mu} M} F^{y M} n_{y}
$$

where ${ }^{5} D_{\hat{\mu}}$ is the covariant derivative with respect to the $5 \mathrm{D}$ metric and $n_{y}=L_{I} / \sqrt{f}$.

The Maxwell equations are given by

$$
\nabla_{N} F^{N M}=0
$$

where $\nabla_{N}$ is the covariant derivative with respect to the $6 \mathrm{D}$ metric.

\section{GRADIENT EXPANSION APPROACH}

We employ the gradient expansion approach to solve the $6 \mathrm{D}$ bulk geometry [19, 20]. Assuming that $\ell$ is not so different from $L_{I}$, the small expansion parameter here is the ratio of the bulk curvature scale to the $4 \mathrm{D}$ intrinsic curvature scale,

$$
\varepsilon=\ell^{2}|R|
$$

1 There we will glue the solutions with different cosmological constants together, and so the subscript $I$ will eventually indicate the different regions of the spacetime. 
The metric and extrinsic curvature are expanded as

$$
\begin{aligned}
\tilde{h}_{\mu \nu} & =h_{\mu \nu}^{(0)}+\varepsilon h_{\mu \nu}^{(1)}+\cdots, & \psi=\psi^{(0)}+\varepsilon \psi^{(1)}+\cdots, \\
K_{\mu} \nu & =\stackrel{(0)}{K_{\mu}} \nu+\varepsilon \stackrel{(1)}{K}_{\mu}^{\nu}+\cdots, & K_{\theta}^{\theta}=\stackrel{(0)}{K_{\theta}^{\theta}}+\varepsilon \stackrel{(1)}{K_{\theta}^{\theta}}+\cdots .
\end{aligned}
$$

We may assume naturally that

$$
b_{\mu}=\varepsilon^{1 / 2} b_{\mu}^{(1 / 2)}+\cdots, \quad K_{\theta}^{\nu}=\varepsilon^{1 / 2} \stackrel{(1 / 2)}{K_{\theta}^{\nu}}+\cdots .
$$

The $(y \theta)$ component of the field strength is expanded as

$$
F_{y \theta}=\stackrel{(0)}{F_{y \theta}}+\varepsilon \stackrel{(1)}{F_{y \theta}}+\cdots
$$

Since $\partial_{\mu} A_{\theta} \sim \varepsilon^{1 / 2} \partial_{y} A_{\theta}$, we have $F_{\mu \theta}=\varepsilon^{1 / 2}{ }^{(1 / 2)} F_{\mu \theta}+\cdots$. We also assume that

$$
F^{\mu y}=\varepsilon^{1 / 2} \stackrel{(1 / 2)}{F}^{\mu y}+\cdots, \quad F_{\mu \nu}=\varepsilon F_{\mu \nu}^{(1)}+\cdots .
$$

Therefore, the $F_{\mu \alpha} F^{\nu \alpha}$ part of the bulk energy-momentum tensor will be higher order in our treatment and it does not contribute to the low energy effective theory. The 5D Ricci tensor is given by

$$
\begin{aligned}
{ }^{5} R_{\mu}^{\nu} & =\varepsilon \frac{1}{a^{2}}\left(R_{\mu}^{\nu}\left[h^{(0)}\right]-\mathcal{D}_{\mu} \mathcal{D}^{\nu} \psi^{(0)}-\mathcal{D}_{\mu} \psi^{(0)} \mathcal{D}^{\nu} \psi^{(0)}\right)+\cdots \\
{ }^{5} R_{\theta}^{\theta} & =-\varepsilon \frac{1}{a^{2}} \mathcal{D}_{\lambda} \mathcal{D}^{\lambda} \psi^{(0)}+\cdots
\end{aligned}
$$

and ${ }^{5} R_{\theta}^{\mu}=\mathcal{O}\left(\varepsilon^{3 / 2}\right),{ }^{2}$ where $R_{\mu}^{\nu}\left[h^{(0)}\right]$ stands for the 4 D Ricci tensor of the zeroth order metric $h_{\mu \nu}^{(0)}$ and $\mathcal{D}_{\mu}$ is the covariant derivative with respect to $h_{\mu \nu}^{(0)}$.

\section{A. Zeroth order equations}

At zeroth order in the gradient expansion, the evolution equations and the Hamiltonian constraint are

$$
\begin{aligned}
& \frac{\sqrt{f}}{L_{I}} \partial_{y} \stackrel{(0)}{K}_{\mu}^{\nu}+\left(\stackrel{(0)}{K_{\lambda}^{\lambda}}+\stackrel{(0)}{K_{\theta}^{\theta}}\right) \stackrel{(0)}{K_{\mu}^{\nu}}=-\frac{1}{4 L_{I}^{2}}\left[1-\frac{1}{\ell^{2} M^{4}}\left(e^{-\psi^{(0)}} \stackrel{(0)}{F_{y \theta}}\right)^{2}\right] \delta_{\mu}^{\nu}, \\
& \frac{\sqrt{f}}{L_{I}} \partial_{y} \stackrel{(0)}{K_{\theta}^{\theta}}+\left(\stackrel{(0)}{K_{\lambda}^{\lambda}}+\stackrel{(0)}{K_{\theta}^{\theta}}\right) \stackrel{(0)}{K_{\theta}^{\theta}}=-\frac{1}{4 L_{I}^{2}}\left[1+\frac{3}{\ell^{2} M^{4}}\left(e^{-\psi^{(0)}} \stackrel{(0)}{F_{y \theta}}\right)^{2}\right] \text {, } \\
& \stackrel{(0)}{K_{\mu}^{\nu}} \stackrel{(0)}{K_{\nu}^{\mu}}-\left(\stackrel{(0)}{K_{\lambda}^{\lambda}}\right)^{2}-2 \stackrel{(0)}{K_{\lambda}^{\lambda}} \stackrel{(0)}{K}_{\theta}^{\theta}=\frac{1}{L_{I}^{2}}\left[1-\frac{1}{\ell^{2} M^{4}}\left(e^{-\psi^{(0)}} \stackrel{(0)}{F_{y \theta}}\right)^{2}\right] \text {. }
\end{aligned}
$$

The Maxwell equations at zeroth order is

$$
\partial_{y}\left(\sqrt{-h^{(0)}} a^{4} e^{-\psi^{(0)}} \stackrel{(0)}{F_{y \theta}}\right)=0 .
$$

The above equations are solved by

$$
\begin{aligned}
& a(y)=y, \\
& f(y)=-\frac{y^{2}}{20}+\frac{\mu}{y^{3}}-\frac{Q^{2}}{12 y^{6}}
\end{aligned}
$$

\footnotetext{
${ }^{2}$ It turns out in Appendix $\mathrm{A}$ that we in fact have ${ }^{5} R_{\theta}^{\mu}=\mathcal{O}\left(\varepsilon^{5 / 2}\right)$.
} 
and

$$
\stackrel{(0)}{F_{y \theta}}=\ell M^{2} \frac{Q}{y^{4}} e^{\psi^{(0)}}, \quad h_{\mu \nu}^{(0)}(x, y)=h_{\mu \nu}(x), \quad \psi^{(0)}(x, y)=\psi^{(0)}(x)
$$

with $\mu$ and $Q$ being constants that characterize the geometry of the internal space.

We assume that the metric function $f(y)$ has two positive roots, $y_{N}$ and $y_{S}\left(<y_{N}\right)$. In other words, we choose the parameters $\mu$ and $Q$ so that $f(y)$ has two positive roots. We impose that $A_{\theta}$ vanishes at these poles, yielding (in the northern half)

$$
A_{\theta}^{(0)}=\frac{\ell M^{2} Q}{3}\left(\frac{1}{y_{N}^{3}}-\frac{1}{y^{3}}\right) e^{\psi^{(0)}}
$$

From this we obtain $\stackrel{(1 / 2)}{F}_{\mu \theta}=\partial_{\mu} \psi^{(0)} \cdot A_{\theta}^{(0)}$. Substituting this into the $\mu$ component of the momentum constraints, we have $\partial_{\mu} \psi^{(0)}=0$. Therefore, $\psi^{(0)}$ must be constant and without loss of generality we may set $\psi^{(0)}=0$. Furthermore, this implies $\stackrel{(1 / 2)}{F_{\mu \theta}}=0$ and hence $F_{\mu \theta}=\mathcal{O}\left(\varepsilon^{3 / 2}\right)+\cdots$.

The above bulk geometry is our "background," which is essentially the double Wick rotated Reissner-Nordström solution [11, 23].

\section{B. First order equations}

Going to first order in the gradient expansion we will obtain the gravitational field equations that govern the behavior of the $4 \mathrm{D}$ metric $h_{\mu \nu}^{(0)}$. The first order equations may contain terms like $\sim \stackrel{(1 / 2)(1 / 2)}{F_{\mu y}} F^{\nu y}$ and $\sim \stackrel{(1 / 2)}{K_{\theta}^{\nu}} K_{\nu}^{\theta}$. However, in Appendix $₫$ we analyze the $\mathcal{O}\left(\varepsilon^{1 / 2}\right)$ equations and show that the gradient expansion of $F_{\mu y}$ and $K_{\theta}{ }^{\nu}$ in fact begins with $\mathcal{O}\left(\varepsilon^{3 / 2}\right)$. Therefore, in the following we will drop the contributions from such terms.

At first order, the $(\mu \nu)$ component of the evolution equations is given by

$$
\frac{\sqrt{f}}{L_{I}}\left[\partial_{y} \stackrel{(1)}{K_{\mu}^{\nu}}+\left(\frac{4}{y}+\frac{\partial_{y} f}{2 f}\right) \stackrel{(1)}{K_{\mu}^{\nu}}+\frac{1}{y}\left(\stackrel{(1)}{K_{\lambda}^{\lambda}}+\stackrel{(1)}{K_{\theta}^{\theta}}\right) \delta_{\mu}^{\nu}\right]=\frac{1}{y^{2}} R_{\mu}^{\nu}+\frac{1}{4} \mathcal{F} \delta_{\mu}^{\nu},
$$

where we defined the useful combination

$$
\mathcal{F}:=\frac{1}{M^{4}}\left(\stackrel{(0)}{F_{y \theta}} F^{y \theta}+\stackrel{(1)}{F_{y \theta}} F^{y \theta}\right)
$$

The 4D Ricci tensor $R_{\mu}^{\nu}$ does not depend on $y$ because it is computed from $h_{\mu \nu}^{(0)}$ which is a function of only $x^{\mu}$. The traceless part of Eq. (20) is found to be

$$
\partial_{y}\left(y^{4} \sqrt{f} \mathbb{K}_{\mu}^{\nu}\right)=y^{2} L_{I} \mathbb{R}_{\mu}^{\nu}
$$

where we defined the traceless part of the relevant tensors as $\mathbb{K}_{\mu}^{\nu}:=\stackrel{(1)}{K_{\mu}^{\nu}}-(1 / 4) \delta_{\mu}^{\nu} \stackrel{(1)}{K_{\lambda}^{\lambda}}$ and $\mathbb{R}_{\mu}^{\nu}:=R_{\mu}^{\nu}-(1 / 4) \delta_{\mu}^{\nu} R$. Eq. (22) can be integrated to give

$$
\mathbb{K}_{\mu}^{\nu}=\frac{1}{3 y \sqrt{f}} L_{I} \mathbb{R}_{\mu}^{\nu}+\frac{1}{y^{4} \sqrt{f}} \mathbb{C}_{\mu}^{\nu}(x)
$$

where the traceless tensor $\mathbb{C}_{\mu}^{\nu}(x)$ is the integration "constant" to be fixed by the boundary conditions.

The trace part of the evolution equations is

$$
\frac{\sqrt{f}}{L_{I}}\left[\partial_{y} \stackrel{(1)}{K}_{\lambda}^{\lambda}+\left(\frac{8}{y}+\frac{\partial_{y} f}{2 f}\right) \stackrel{(1)}{K_{\lambda}^{\lambda}}+\frac{4}{y} \stackrel{(1)}{K_{\theta}^{\theta}}\right]=\frac{1}{y^{2}} R+\mathcal{F},
$$

and the $(\theta \theta)$ component of the evolution equations is

$$
\frac{\sqrt{f}}{L_{I}}\left[\partial_{y} \stackrel{(1)}{K_{\theta}^{\theta}}+\left(\frac{4}{y}+\frac{\partial_{y} f}{f}\right) \stackrel{(1)}{K_{\theta}^{\theta}}+\frac{\partial_{y} f}{2 f} \stackrel{(1)}{K_{\lambda}^{\lambda}}\right]=-\frac{3}{4} \mathcal{F} .
$$


The Hamiltonian constraint at first order reduces to

$$
\frac{1}{y^{2}} R+\mathcal{F}=2 \frac{\sqrt{f}}{L_{I}}\left[\left(\frac{3}{y}+\frac{\partial_{y} f}{2 f}\right) \stackrel{(1)}{K_{\lambda}^{\lambda}}+\frac{4}{y} \stackrel{(1)}{K}_{\theta}^{\theta}\right]
$$

It is possible to solve the set of equations (24)-(26) and find the bulk profile of the extrinsic curvature and $\mathcal{F}$ (see Appendix [B]. Then, with the help of the relation

$$
\stackrel{(1)}{F_{y \theta}}=\ell^{2} L_{I}^{2}\left(2 \psi^{(1)} \stackrel{(0)}{F}^{y \theta}+{\stackrel{(1)}{F^{y} \theta}}^{(1)},\right.
$$

one finds the bulk profile of the gauge field. Equivalently, one can use the first order Maxwell equations,

$$
\partial_{y}\left(y^{4} \stackrel{(1)}{F^{y \theta}}\right)+\frac{L_{I}}{\sqrt{f}} y^{4} \stackrel{(0)}{F}^{\theta}\left(\stackrel{(1)}{K_{\lambda}^{\lambda}}+\stackrel{(1)}{K_{\theta}^{\theta}}\right)=0
$$

to solve for the gauge field. As will be seen, however, we do not need the explicit form of the solution for the extrinsic curvature and gauge field in order to derive an effective theory on the brane; it suffices to solve the bulk evolution of the combination of first order variables

$$
\mathcal{K}:=\frac{3}{4} \stackrel{(1)}{K}_{\lambda}^{\lambda}+{\stackrel{(1)}{K_{\theta}^{\theta}}}^{\theta}+\frac{1}{M^{4}} \frac{L_{I}}{\sqrt{f}} \stackrel{(0)}{y \theta}^{(1)} A_{\theta}^{(1)}+\frac{\sqrt{f}}{L_{I}}\left(\frac{\partial_{y} f}{2 f}-\frac{1}{y}\right) \psi^{(1)} .
$$

The evolution equation for $\mathcal{K}$ can be obtained as follows. Using the relation (27), it is straightforward to show

$$
\partial_{y}\left(y^{4} \sqrt{f} \mathcal{K}\right)=\partial_{y}\left[y^{4} \sqrt{f}\left(\frac{3}{4} \stackrel{(1)}{K}_{\lambda}^{\lambda}+\stackrel{(1)}{K}_{\theta}^{\theta}\right)\right]+y^{4} \sqrt{f}\left(\frac{\partial_{y} f}{2 f}-\frac{1}{y}\right) \stackrel{(1)}{K_{\theta}^{\theta}}+\frac{1}{2} y^{4} L_{I} \mathcal{F} .
$$

Then, using the evolution equations (24), (25), and the Hamiltonian constraint (26), we arrive at

$$
\partial_{y}\left(y^{4} \sqrt{f} \mathcal{K}\right)=\frac{y^{2}}{4} L_{I} R
$$

The structure of Eq. (31) is identical to the traceless equations (22), and the general solution is given by

$$
\mathcal{K}=\frac{1}{12 y \sqrt{f}} L_{I} R+\frac{1}{y^{4} \sqrt{f}} \chi(x),
$$

where $\chi(x)$ is an integration "constant" to be determined by the boundary conditions.

The $\mu$ component of the momentum constraints at first order is given by

$$
\mathcal{D}_{\nu} \mathbb{K}_{\mu}^{\nu}-\mathcal{D}_{\mu}\left(\frac{3}{4}{\stackrel{(1)}{K_{\lambda}}}^{\lambda}+\stackrel{(1)}{K_{\theta}^{\theta}}\right)+\frac{\sqrt{f}}{L_{I}}\left(\frac{1}{y}-\frac{\partial_{y} f}{2 f}\right) \mathcal{D}_{\mu} \stackrel{(1)}{\psi}=\frac{1}{M^{4}} \mathcal{D}_{\mu} \stackrel{(1)}{A_{\theta}} \stackrel{(0)}{F^{y \theta}} \frac{L_{I}}{\sqrt{f}}
$$

which can be simplified using $\mathcal{K}$ to

$$
\mathcal{D}_{\nu} \mathbb{K}_{\mu}^{\nu}-\mathcal{D}_{\mu} \mathcal{K}=0
$$

Eq. (33) and the Bianchi identity, $\mathcal{D}_{\nu}\left[R_{\mu}^{\nu}-(1 / 2) \delta_{\mu}^{\nu} R\right]=0$, imply the constraint for the integration constants:

$$
\mathcal{D}_{\nu} \mathbb{C}_{\mu}^{\nu}-\mathcal{D}_{\mu} \chi=0
$$

\section{REPLACING CONICAL BRANES WITH REGULARIZED 4-BRANES}

The metric function $f(y)$ vanishes at $y_{N}$ and $y_{S}$. These points develop conical singularities in general and they are regarded as source 3-branes. To resolve the singularities, we replace each of the conical branes with a cylindrical codimension-one brane and fill in the interior with a regular cap [8, 9]. The geometries of the two caps and central bulk region are described by the $6 \mathrm{D}$ solutions obtained in the previous section with different cosmological constants 
$\left(L_{+}\left(L_{-}\right)\right.$for the north (south) cap and $L_{0}$ for the central bulk). Near the pole $y=y_{p}(p=N, S)$, we have $\left.f(y) \simeq \partial_{y} f\right|_{y=y_{p}}\left(y-y_{p}\right)$. In order for the cap to close regularly at $y_{p}$, we impose

$$
\left.\frac{\ell}{L_{I}} \frac{\left|\partial_{y} f\right|}{2} e^{\psi}\right|_{y=y_{p}}=2 \pi
$$

Clearly, it is required that $\psi\left(x, y_{p}\right)=$ constant. Without loss of generality we can set this constant contribution to be zero. In particular, we have $\psi^{(1)} \lesssim\left(y-y_{p}\right)$ near $y=y_{p}$.

We consider the following action for each 4 -brane [8]:

$$
S^{ \pm}=-\int d^{5} x \sqrt{-q}\left[\lambda_{ \pm}+\frac{v_{ \pm}^{2}}{2} q^{\hat{\mu} \hat{\nu}}\left(\partial_{\hat{\mu}} \Sigma_{ \pm}-\mathrm{e} A_{\hat{\mu}}\right)\left(\partial_{\hat{\nu}} \Sigma_{ \pm}-\mathrm{e} A_{\hat{\nu}}\right)\right]+S_{m}^{ \pm}
$$

where $\lambda_{ \pm}$is the brane tension, $v_{ \pm}$is the vacuum expectation value of the brane Higgs field and $\Sigma_{ \pm}$is its Goldstone mode. $S_{m}^{ \pm}$represents the matter action on the brane. The brane action necessarily couples to $A_{\hat{\mu}}$ in order to account for the jump of the Maxwell field at the brane. This implies that the brane location is fixed[see Eq. (39) below]. The total brane energy-momentum tensor derived from the above action is

$$
T_{\hat{\mu}(t o t)}^{ \pm \hat{\nu}}=-\lambda_{ \pm} \delta_{\hat{\mu}}^{\hat{\nu}}+v_{ \pm}^{2}\left[\left(\partial_{\hat{\mu}} \Sigma_{ \pm}-\mathrm{e} A_{\hat{\mu}}\right)\left(\partial^{\hat{\nu}} \Sigma_{ \pm}-\mathrm{e} A^{\hat{\nu}}\right)-\frac{1}{2}\left(\partial_{\hat{\lambda}} \Sigma_{ \pm}-\mathrm{e} A_{\hat{\lambda}}\right)\left(\partial^{\hat{\lambda}} \Sigma_{ \pm}-\mathrm{e} A^{\hat{\lambda}}\right) \delta_{\hat{\mu}}^{\hat{\nu}}\right]+T_{\hat{\mu}}^{ \pm \hat{\nu}},
$$

where $T_{\hat{\mu}}^{ \pm \hat{\nu}}$ is the energy-momentum tensor of the matter fields on the branes. We assume that $T_{\mu}^{ \pm \theta}=T_{\theta}^{ \pm \nu}=0$.

Each of the caps and the central bulk spacetime are glued together so as to satisfy the Israel conditions [24] and the jump conditions for the Maxwell field. From now on we will suppress the index \pm if it is not necessary. We start with assuming that the brane location is given by a $x$-dependent function $y=\varphi(x)$. The brane induced metric is given by $q_{\hat{\mu} \hat{\nu}} d x^{\hat{\mu}} d x^{\hat{\nu}}=\ell^{2} f(\varphi(x)) d \theta^{2}+\varphi^{2}(x) h_{\mu \nu}^{(0)} d x^{\mu} d x^{\nu}+\mathcal{O}(\varepsilon)$. The equation of motion for each scalar field $\Sigma_{ \pm}$at zeroth order reduces to $\partial_{\theta}^{2} \Sigma_{ \pm}^{(0)}=0$, and hence

$$
\Sigma_{ \pm}^{(0)}=n_{ \pm} \theta+\sigma_{ \pm}^{(0)}(x) .
$$

Here, $n_{ \pm}$must be integer because $\Sigma_{ \pm}$is the phase of the Higgs field and so $e^{i \Sigma(\theta+2 \pi, x)}=e^{i \Sigma(\theta, x)}$. This property should hold at any order in the gradient expansion. Therefore, the solution for $\Sigma$ including higher order corrections should be of the form $\Sigma=n \theta+\sigma^{(0)}(x)+\varepsilon \sigma^{(1)}(x)+\cdots$.

We shall show now that the brane location is in fact independent of $x$. To do so, let us consider the $\theta$ component of the Maxwell jump conditions at zeroth order:

$$
\left[\left[\bar{n}^{M} F_{M \theta}^{(0)}\right]\right]=-\mathrm{e} v^{2}\left(\partial_{\theta} \Sigma^{(0)}-\mathrm{e} A_{\theta}^{(0)}\right)
$$

where $[[A]]:=\left.A\right|_{y=\varphi+\epsilon}-\left.A\right|_{y=\varphi-\epsilon}$ and $\bar{n}^{M}$ is the unit normal to the brane. At this order, we have $\bar{n}^{M} \simeq\left(n^{y}, 0\right)$. The right hand side is independent of $x$ and hence the brane location must be $y=$ constant $\left(=: y_{ \pm}\right)$. The Israel conditions are given by

$$
\begin{aligned}
{\left[\left[\frac{\sqrt{f}}{L_{I}}\left(\frac{3}{y}+\frac{\partial_{y} f}{2 f}\right)\right]\right] } & =-\frac{\lambda}{M^{4}}-\frac{v^{2}}{2 M^{4}}\left(\partial_{\theta} \Sigma^{(0)}-\mathrm{e} A_{\theta}^{(0)}\right)\left(\partial^{\theta} \Sigma^{(0)}-\mathrm{e} A^{\theta(0)}\right)=T_{\mu(t o t)}^{\nu(0)}, \\
{\left[\left[\frac{\sqrt{f}}{L_{I}} \frac{4}{y}\right]\right] } & =-\frac{\lambda}{M^{4}}+\frac{v^{2}}{2 M^{4}}\left(\partial_{\theta} \Sigma^{(0)}-\mathrm{e} A_{\theta}^{(0)}\right)\left(\partial^{\theta} \Sigma^{(0)}-\mathrm{e} A^{\theta(0)}\right)=T_{\theta(t o t)}^{\theta(0)} .
\end{aligned}
$$

The above conditions (39)-(41) determine the brane location $y_{ \pm}$and the parameter of the solution $n_{ \pm}$. For the current purpose we need no further detail; see Sec. II of Ref. [17] for more explanation of the configuration of the branes.

\section{EFFECTIVE THEORY ON A REGULARIZED BRANE}

We go on to specifying the first order boundary conditions at the poles and branes. As to the regularity conditions at the poles, it is required that

$$
\mathbb{K}_{\mu}^{\nu}, \stackrel{(1)}{K_{\lambda}^{\lambda}}, \stackrel{(1)}{K_{\theta}^{\theta}} \lesssim\left|y-y_{p}\right|^{1 / 2}
$$


With this, the evolution equations for the extrinsic curvature (22), (24), and (25) are regular at the poles. We also require that $\left|\stackrel{(1)}{F_{y \theta}}\right|<\infty$ at $y=y_{p}$. This implies that $A_{\theta}^{(1)} \lesssim\left(y-y_{p}\right)$ near the pole. Noting that $\psi^{(1)} \lesssim\left(y-y_{p}\right)$, we have $\mathcal{K} \lesssim\left|y-y_{p}\right|^{1 / 2}$ near $y=y_{p}$.

The $(\mu \nu)$ component of the Israel conditions at first order are given by

$$
\left[\left[\stackrel{(1)}{K_{\mu}^{\nu}}-\delta_{\mu}^{\nu}\left({\stackrel{(1)}{K_{\lambda}^{\lambda}}}^{\lambda}+\stackrel{(1)}{K_{\theta}^{\theta}}\right)\right]\right]=-\frac{T_{\mu}^{\nu}}{M^{4}}-\frac{v^{2}}{M^{4}} \frac{1}{\ell^{2} f}\left[\left(\partial_{\theta} \Sigma^{(0)}-\mathrm{e} A_{\theta}^{(0)}\right) \mathrm{e} A_{\theta}^{(1)}+\left(\partial_{\theta} \Sigma^{(0)}-\mathrm{e} A_{\theta}^{(0)}\right)^{2} \psi^{(1)}\right] \delta_{\mu}^{\nu} .
$$

In deriving this we used $\left(\partial^{\nu} \Sigma-\mathrm{e} A^{\nu}\right)^{(1 / 2)}=0$ which is shown in Appendix $\mathrm{A}$ The traceless part of Eq. (43) reads

$$
\left[\left[\mathbb{K}_{\mu}^{\nu}\right]\right]=-\frac{1}{M^{4}} \mathbb{T}_{\mu}^{\nu}
$$

where $\mathbb{T}_{\mu}^{\nu}$ is the traceless part of the energy-momentum tensor. Noting that ${ }^{3}$

$$
[[\mathcal{K}]]=\frac{1}{4}\left[\left[3 \stackrel{(1)}{K_{\lambda}^{\lambda}}+4 \stackrel{(1)}{K_{\theta}^{\theta}}\right]\right]+\frac{1}{M^{4}} \frac{1}{\ell^{2} f}\left[\left[n^{y} \stackrel{(0)}{F_{y \theta}}\right]\right] A_{\theta}^{(1)}+\left[\left[\frac{\sqrt{f}}{L_{I}}\left(\frac{\partial_{y} f}{2 f}-\frac{1}{y}\right)\right]\right] \psi^{(1)},
$$

and using the zeroth order Israel conditions and jump conditions for the Maxwell field, we find that the trace part of Eq. (43) yields

$$
[[\mathcal{K}]]=\frac{1}{4 M^{4}} T_{\lambda}^{\lambda}
$$

The junction conditions (44) and (45) together with the momentum constraint (33) imply the local conservation law for the energy-momentum tensor of brane localized matter:

$$
\mathcal{D}_{\nu} T_{\mu}^{\nu}=0 .
$$

Now we are to fix the integration constants. From the regularity condition at the north pole one can determine the integration constant in the north cap and we have

$$
\mathbb{K}_{\mu}^{\nu}=\frac{y^{3}-y_{N}^{3}}{3 y^{4} \sqrt{f}} L_{+} \mathbb{R}_{\mu}^{\nu} \quad\left(y_{+}<y \leq y_{N}\right) .
$$

Then, using the Israel conditions at the brane we obtain

$$
\mathbb{K}_{\mu}^{\nu}=\frac{1}{3 y \sqrt{f}} L_{0} \mathbb{R}_{\mu}^{\nu}+\frac{1}{y^{4} \sqrt{f}} \mathbb{C}_{\mu}^{\nu} \quad\left(y_{-}<y<y_{+}\right),
$$

where

$$
\mathbb{C}_{\mu}^{\nu}=-\frac{\left(y_{N}^{2}-y_{+}^{3}\right) L_{+}+y_{+}^{3} L_{0}}{3} \mathbb{R}_{\mu}^{\nu}+y_{+}^{4} \sqrt{f_{+}} \frac{\mathbb{T}_{\mu}^{+\nu}}{M^{4}}
$$

and $f_{+}:=f\left(y_{+}\right)$. The regularity at the south pole determines the extrinsic curvature in the south cap as

$$
\mathbb{K}_{\mu}^{\nu}=\frac{y^{3}-y_{S}^{3}}{3 y^{4} \sqrt{f}} L_{-} \mathbb{R}_{\mu}^{\nu} \quad\left(y_{S} \leq y<y_{-}\right) .
$$

The Israel conditions at $y=y_{-}$imply

$$
\frac{\left(y_{-}^{3}-y_{S}^{3}\right) L_{-}-y_{-}^{3} L_{0}}{3} \mathbb{R}_{\mu}^{\nu}-\mathbb{C}_{\mu}^{\nu}=y_{-}^{4} \sqrt{f_{-}} \frac{\mathbb{T}_{\mu}^{-\nu}}{M^{4}}
$$

\footnotetext{
3 The gauge field $A_{\theta}^{(1)}$ is continuous across the brane in order for the brane action to be well-defined. The continuity of the induced metric imposes that $\psi^{(1)}$ is continuous across the brane.
} 
which is rearranged to give

$$
\frac{\ell_{*}^{2}}{\ell} \mathbb{R}_{\mu}^{\nu}=\frac{1}{M^{4}}\left(y_{+}^{4} \sqrt{f_{+}} \mathbb{T}_{\mu}^{+\nu}+y_{-}^{4} \sqrt{f_{-}} \mathbb{T}_{\mu}^{-\nu}\right)
$$

where

$$
\ell_{*}^{2}:=\ell \int_{y_{S}}^{y_{N}} L_{I} y^{2} d y
$$

and $f_{-}:=f\left(y_{-}\right)$. Obviously, for the trace part we have the same relation as (52) with the substitution $\mathbb{R}_{\mu}^{\nu} \rightarrow R / 4$ and $\mathbb{T}_{\mu}^{ \pm \nu} \rightarrow-T_{\lambda}^{ \pm \lambda} / 4$ (and thus the constraint for the integration constants (34) is trivially satisfied). Taking into account that the induced metric on the north brane is given by $y_{+}^{2} h_{\mu \nu}$, we define the brane Ricci tensor as $\mathcal{R}_{\mu}^{\nu}:=R_{\mu}^{\nu} / y_{+}^{2}$. Combining the traceless and trace equations, we finally arrive at

$$
\mathcal{R}_{\mu}^{\nu}-\frac{1}{2} \delta_{\mu}^{\nu} \mathcal{R}=\kappa_{+}^{2} \bar{T}_{\mu}^{+\nu}+\frac{y_{-}^{2}}{y_{+}^{2}} \kappa_{-}^{2} \bar{T}_{\mu}^{-\nu},
$$

where we defined the $4 \mathrm{D}$ gravitational coupling at each brane as

$$
\kappa_{ \pm}^{2}:=\frac{y_{ \pm}^{2}}{2 \pi \ell_{*}^{2} M^{4}},
$$

and the energy-momentum tensor integrated along the $\theta$-direction as $\bar{T}_{\mu}^{ \pm \nu}:=2 \pi \ell \sqrt{f_{ \pm}} T_{\mu}^{ \pm \nu}$. Eq. (54) shows that gravity at low energies is described by general relativity (when matter on the south brane can be neglected.) This generalizes the perturbative analysis of [8, 17] to the nonlinear regime. The effect of the extra scalar mode that appears in [8, 17] is higher order in the gradient expansion and hence is not observed in the present leading order analysis.

\section{CONCLUSIONS}

In this paper we have considered six-dimensional Einstein-Maxwell model of warped braneworlds, where the extra dimensions are stabilized by a flux. Conical singularities in such models can be regularized by replacing the 3-branes with codimension-one branes. Using the gradient expansion approach, we have derived a low energy effective theory on the regularized brane, and have shown that standard 4D Einstein gravity is reproduced on the brane. Though the present analysis is restricted up to first order, one can go on to the higher order expansions to include the effect of Kaluza-Klein modes. The scalar zero mode corresponding to the fluctuation of the internal space volume would also appear at second order. Computing higher order corrections would be interesting and is one of the remaining issues.

Our result has been derived without relying on linear perturbations in the Minkowski brane background. The effective Einstein equations (54) are valid even in the nonlinear regime, and hence can be applied to the cosmological dynamics at low energies (i.e., to late-time cosmology in which the Hubble horizon is much larger than the compactification scale). Since the effective theory coincides with $4 \mathrm{D}$ general relativity, the present model can reproduce conventional cosmology on the brane. Recently, cosmology on a moving brane in a given (static) background has been investigated in the context of regularized braneworlds with flux compactifications [25, 26]. Their results are not compatible with the realistic cosmological evolution, suggesting that the static bulk assumption is an oversimplification. On the other hand, we have started with a more general metric ansatz (2) whose internal space component is nontrivially dependent on the external spacetime coordinates. Thus, constructing explicit cosmological solutions in the $6 \mathrm{D}$ flux compactification model is not so simple as in the 5D Randall-Sundrum-type braneworlds (see, however, [27, 28]).

$6 \mathrm{D}$ braneworlds are often discussed not only in a simplified Einstein-Maxwell setup but also in the context of gauged chiral supergravity [29, 30]. Supergravity models generally have conical brane singularities too, and the regularization procedure has been proposed in [9, 31]. The present gradient expansion approach is useful for the supergravity braneworlds as well.

\section{Acknowledgments}

SF and TK are supported by the JSPS under Contract Nos. 19-2570 and 19-4199. TS is supported by Grant-Aid for Scientific Research from Ministry of Education, Science, Sports and Culture of Japan (Nos. 17740136, 17340075, and 19GS0219), the Japan-U.K., Japan-France and Japan-India Research Cooperative Programs. 


\section{APPENDIX A: $\mathcal{O}\left(\varepsilon^{1 / 2}\right)$ QUANTITIES}

Starting with the assumptions (8) and (9), we shall show in this appendix that the leading order terms in the gradient expansion of $K_{\theta}^{\nu}$ and $F^{\mu y}$ are in fact $\mathcal{O}\left(\varepsilon^{3 / 2}\right)$.

The $\mu$ component of the $\mathcal{O}\left(\varepsilon^{1 / 2}\right)$ Maxwell equations reads

$$
\partial_{y}\left(y^{4} \stackrel{(1 / 2)}{F^{y \mu}}\right)=0
$$

and thus we have

$$
\stackrel{(1 / 2)}{F^{\mu y}}=M^{2} \frac{C_{1}^{\mu}(x)}{y^{4}}
$$

The $\mathcal{O}\left(\varepsilon^{1 / 2}\right)$ evolution equation reduces to

$$
\begin{aligned}
\frac{1}{L_{I}} \frac{1}{y^{4}} \partial_{y}\left(y^{4} \sqrt{f} \stackrel{(1 / 2)}{K_{\theta}^{\nu}}\right) & =-\frac{1}{M^{4}} \stackrel{(0)}{F}_{\theta y} \stackrel{(1 / 2)}{F}^{\nu y} \\
& =\ell Q \frac{C_{1}^{\nu}(x)}{y^{8}}
\end{aligned}
$$

which can be integrated to give

$$
\stackrel{(1 / 2)}{K_{\theta}^{\nu}}=\frac{1}{y^{4} \sqrt{f}}\left[-\frac{L_{I} \ell Q}{3} \frac{C_{1}^{\nu}(x)}{y^{3}}+C_{2}^{\nu}(x)\right] .
$$

The $\mathcal{O}(\varepsilon)$ evolution equations contain terms like $\stackrel{(1 / 2)(1 / 2)}{F_{\mu y}} F^{\nu y} \propto h_{\mu \lambda} C_{1}^{\lambda} C_{1}^{\nu} /\left[y^{6} f(y)\right]$. Thus, in the cap regions it is required that $C_{1}^{\nu}=0$ because otherwise this term would show a singular behavior at the poles. Similarly, to ensure the regular

behavior of $\stackrel{(1 / 2)}{K}_{\theta}^{\nu}$ at the poles we impose $C_{2}^{\nu}=0$ in the cap regions. To fix the integration constants in the central bulk region, we invoke the junction conditions at the branes. The jump conditions of the Maxwell field and Israel conditions imply, respectively,

$$
\begin{aligned}
{\left[\left[n_{y} \stackrel{(1 / 2)}{F^{y \mu}}\right]\right] } & =-\mathrm{e} v^{2}\left(\partial^{\mu} \Sigma-\mathrm{e} A^{\mu}\right)^{(1 / 2)} \\
{\left[\left[\begin{array}{c}
(1 / 2) \\
K_{\theta}^{\mu}
\end{array}\right]\right] } & =-\frac{v^{2}}{M^{4}}\left(\partial_{\theta} \Sigma^{(0)}-\mathrm{e} A_{\theta}^{(0)}\right)\left(\partial^{\mu} \Sigma-\mathrm{e} A^{\mu}\right)^{(1 / 2)}
\end{aligned}
$$

Combining these two equations and noting that $\stackrel{(1 / 2)}{K}_{\theta}^{\mu}=0=\stackrel{(1 / 2)}{F^{y \mu}}$ in each cap, we obtain two linear algebraic equations for $C_{1}^{\nu}$ and $C_{2}^{\nu}$ :

$$
\left.\left[\stackrel{(1 / 2)}{K_{\theta}^{\mu}}-\frac{1}{\mathrm{e} M^{4}}\left(\partial_{\theta} \Sigma^{(0)}-\mathrm{e} A_{\theta}^{(0)}\right) n_{y} \stackrel{(1 / 2)}{F}^{y \mu}\right]\right|_{y=y_{ \pm} \mp \epsilon}=0
$$

Thus, it is now clear that both $C_{1}^{\nu}$ and $C_{2}^{\nu}$ vanish in the bulk.

To sum up, we have shown in this appendix that

$$
\begin{aligned}
& K_{\theta}^{\mu}=\varepsilon^{3 / 2} \stackrel{(3 / 2)}{K_{\theta}^{\mu}}+\cdots, \\
& F^{y \mu}=\varepsilon^{3 / 2} \stackrel{(3 / 2)}{F^{y \mu}}+\cdots,
\end{aligned}
$$

and that

$$
\left(\partial^{\mu} \Sigma-\mathrm{e} A^{\mu}\right)^{(1 / 2)}=0 \quad \text { on the branes. }
$$




\section{APPENDIX B: SOLVING THE FIRST ORDER EVOLUTION EQUATIONS AND HAMILTONIAN CONSTRAINT}

For completeness we present here how we can solve the evolution equations (24), (25), and the Hamiltonian constraint (26) analytically. Eliminating $\mathcal{F}$, we obtain the coupled evolution equations for ${\stackrel{(1)}{K_{\lambda}}}^{\lambda}$ and $\stackrel{(1)}{K_{\theta}^{\theta}}$ :

$$
\begin{aligned}
& \partial_{y}{\stackrel{(1)}{K_{\lambda}}}^{\lambda}+\left(\frac{2}{y}-\frac{\partial_{y} f}{2 f}\right){\stackrel{(1)}{K_{\lambda}^{\lambda}}}^{\lambda}-\frac{4}{y}{\stackrel{(1)}{K_{\theta}^{\theta}}}^{\theta}=0, \\
& \partial_{y} \stackrel{(1)}{K}_{\theta}^{\theta}+\left(\frac{10}{y}+\frac{\partial_{y} f}{f}\right) \stackrel{(1)}{K_{\theta}^{\theta}}+\left(\frac{9}{2 y}+\frac{5 \partial_{y} f}{4 f}\right) \stackrel{(1)}{K_{\lambda}^{\lambda}}=\frac{3}{4 y^{2}} \frac{L_{I}}{\sqrt{f}} R \text {. }
\end{aligned}
$$

Then, we substitute Eq. (B1) into Eq. (B) to obtain the single second order differential equation for $K_{\lambda}^{\lambda}$. In terms of

$$
\Omega:=\frac{\stackrel{(1)}{K}_{\lambda}^{\lambda}}{\sqrt{f} \partial_{y}(\sqrt{f} / y)}
$$

the equation can be written in a simple form:

$$
\partial_{y}^{2} \Omega-\frac{\partial_{y}^{2} \mathcal{I}}{\partial_{y} \mathcal{I}} \partial_{y} \Omega=\frac{3}{y^{3} f \partial_{y}(\sqrt{f} / y)} L_{I} R
$$

where

$$
\partial_{y} \mathcal{I}(y):=\frac{1}{y^{16}(\sqrt{f} / y)^{3}\left[\partial_{y}(\sqrt{f} / y)\right]^{2}}
$$

The general solution to Eq. (B4) is given by

$$
\Omega=C_{1}(x)+C_{2}(x) \mathcal{I}(y)+3 L_{I} R \int^{y} d \bar{y} \partial_{\bar{y}} \mathcal{I}(\bar{y}) \int^{\bar{y}} \frac{d \tilde{y}}{\tilde{y}^{3} f \partial_{\tilde{y}}(\sqrt{f} / \tilde{y}) \partial_{\tilde{y}} \mathcal{I}(\tilde{y})}
$$

Eqs. (B1) and (B3) allow one to write the extrinsic curvature in terms of $\Omega$. Then $\mathcal{F}$ can also be written in terms of $\Omega$ using the Hamiltonian constraint.

[1] L. Randall and R. Sundrum, Phys. Rev. Lett. 83, 3370 (1999) arXiv:hep-ph/9905221]; L. Randall and R. Sundrum, Phys. Rev. Lett. 83, 4690 (1999) arXiv:hep-th/9906064.

[2] R. Maartens, Living Rev. Rel. 7, 7 (2004) arXiv:gr-qc/0312059; P. Brax, C. van de Bruck and A. C. Davis, Rept. Prog. Phys. 67, 2183 (2004) arXiv:hep-th/0404011 ; C. Csaki, arXiv:hep-ph/0404096

[3] S. Kachru, R. Kallosh, A. Linde and S. P. Trivedi, Phys. Rev. D 68, 046005 (2003) arXiv:hep-th/0301240; S. Kachru, R. Kallosh, A. Linde, J. M. Maldacena, L. McAllister and S. P. Trivedi, JCAP 0310, 013 (2003) arXiv:hep-th/0308055.

[4] M. R. Douglas and S. Kachru, Rev. Mod. Phys. 79, 733 (2007) arXiv:hep-th/0610102.

[5] J. W. Chen, M. A. Luty and E. Ponton, JHEP 0009, 012 (2000) arXiv:hep-th/0003067] S. M. Carroll and M. M. Guica, arXiv:hep-th/0302067; I. Navarro, JCAP 0309, 004 (2003) arXiv:hep-th/0302129; Y. Aghababaie, C. P. Burgess, S. L. Parameswaran and F. Quevedo, Nucl. Phys. B 680, 389 (2004) arXiv:hep-th/0304256].

[6] I. Navarro, Class. Quant. Grav. 20, 3603 (2003) arXiv:hep-th/0305014]; H. P. Nilles, A. Papazoglou and G. Tasinato, Nucl. Phys. B 677, 405 (2004) arXiv:hep-th/0309042; H. M. Lee, Phys. Lett. B 587, 117 (2004) arXiv:hep-th/0309050; J. Vinet and J. M. Cline, Phys. Rev. D 70, 083514 (2004) arXiv:hep-th/0406141; ;. Garriga and M. Porrati, JHEP 0408, 028 (2004) arXiv:hep-th/0406158; J. Vinet and J. M. Cline, Phys. Rev. D 71, 064011 (2005) arXiv:hep-th/0501098.

[7] K. Koyama, arXiv:0706.1557] [astro-ph].

[8] M. Peloso, L. Sorbo and G. Tasinato, Phys. Rev. D 73, 104025 (2006) arXiv:hep-th/0603026.

[9] E. Papantonopoulos, A. Papazoglou and V. Zamarias, JHEP 0703, 002 (2007) arXiv:hep-th/0611311.

[10] B. Himmetoglu and M. Peloso, Nucl. Phys. B 773, 84 (2007) arXiv:hep-th/0612140.

[11] J. Louko and D. L. Wiltshire, JHEP 0202, 007 (2002) arXiv:hep-th/0109099.

[12] B. M. N. Carter, A. B. Nielsen and D. L. Wiltshire, JHEP 0607, 034 (2006) arXiv:hep-th/0602086].

[13] T. Kobayashi and Y. i. Takamizu, arXiv:0707.0894 [hep-th]. 
[14] S. Kanno, D. Langlois, M. Sasaki and J. Soda, arXiv:0707.4510 [hep-th].

[15] S. A. Appleby and R. A. Battye, arXiv:0707.4238 [hep-ph].

[16] N. Kaloper and D. Kiley, JHEP 0603, 077 (2006) arXiv:hep-th/0601110]; D. Kiley, arXiv:0708.1016 [hep-th].

[17] T. Kobayashi and M. Minamitsuji, Phys. Rev. D 75, 104013 (2007) arXiv:hep-th/0703029.

[18] T. Shiromizu, K. i. Maeda and M. Sasaki, Phys. Rev. D 62, 024012 (2000) arXiv:gr-qc/9910076.

[19] S. Kanno and J. Soda, Phys. Rev. D 66, 043526 (2002) arXiv:hep-th/0205188.

[20] T. Shiromizu and K. Koyama, Phys. Rev. D 67, 084022 (2003) arXiv:hep-th/0210066].

[21] T. Shiromizu, K. Koyama, S. Onda and T. Torii, Phys. Rev. D 68, 063506 (2003) arXiv:hep-th/0305253; S. Onda, T. Shiromizu, K. Koyama and S. Hayakawa, Phys. Rev. D 69, 123503 (2004) arXiv:hep-th/0311262 ; K. Koyama, K. Koyama and F. Arroja, Phys. Lett. B 641, 81 (2006) arXiv:hep-th/0607145]; T. Kobayashi, T. Shiromizu and N. Deruelle, Phys. Rev. D 74, 104031 (2006) arXiv:hep-th/0608166.

[22] D. Yamauchi and M. Sasaki, arXiv:0705.2443 [gr-qc].

[23] G. W. Gibbons and D. L. Wiltshire, Nucl. Phys. B 287, 717 (1987) arXiv:hep-th/0109093]; S. Mukohyama, Y. Sendouda, H. Yoshiguchi and S. Kinoshita, JCAP 0507, 013 (2005) arXiv:hep-th/0506050.

[24] W. Israel, Nuovo Cim. B 44S10, 1 (1966) [Erratum-ibid. B 48, 463 (1967 NUCIA,B44,1.1966)].

[25] E. Papantonopoulos, A. Papazoglou and V. Zamarias, arXiv:0707.1396 [hep-th].

[26] M. Minamitsuji and D. Langlois, arXiv:0707.1426 [hep-th].

[27] J. M. Cline, J. Descheneau, M. Giovannini and J. Vinet, JHEP 0306, 048 (2003) arXiv:hep-th/0304147].

[28] A. J. Tolley, C. P. Burgess, C. de Rham and D. Hoover, New J. Phys. 8, 324 (2006) arXiv:hep-th/0608083; T. Kobayashi and M. Minamitsuji, arXiv:0705.3500 [hep-th]; E. J. Copeland and O. Seto, arXiv:0705.4169 [hep-th].

[29] G. W. Gibbons, R. Guven and C. N. Pope, Phys. Lett. B 595, 498 (2004) arXiv:hep-th/0307238; Y. Aghababaie et al., JHEP 0309, 037 (2003) arXiv:hep-th/0308064; C. P. Burgess, F. Quevedo, G. Tasinato and I. Zavala, JHEP 0411, 069 (2004) arXiv:hep-th/0408109; A. J. Tolley, C. P. Burgess, D. Hoover and Y. Aghababaie, JHEP 0603, 091 (2006) arXiv:hep-th/0512218.

[30] H. M. Lee and A. Papazoglou, Nucl. Phys. B 747, 294 (2006) [Erratum-ibid. B 765, 200 (2007)] arXiv:hep-th/0602208; C. P. Burgess, C. de Rham, D. Hoover, D. Mason and A. J. Tolley, JCAP 0702, 009 (2007) arXiv:hep-th/0610078.

[31] C. P. Burgess, D. Hoover and G. Tasinato, arXiv:0705.3212 [hep-th]. 